\title{
Late onset Multiple Sclerosis: A More Divergent Clinical Spectrum than Commonly Perceived
}

\author{
Naveed Iqbal* \\ Department of Neurology, Khoula Hospital, Ministry of Health, Muscat
}

Submission: May 30,2019; Published: June 14, 2019

*Corresponding author: Naveed Iqbal, Department of Neurology, Khoula Hospital, Ministry of Health, Sultanate of Oman, Muscat

\begin{abstract}
Multiple Sclerosis (MS) is an autoimmune disorder of the central nervous system, which affects young people. A large percentage of the patients falls between the ages of 30 and 50 years. Less frequently, MS is first diagnosed after the age of 50 , which is by definition called Late Onset Multiple Sclerosis (LOMS) [1-4] Roughly, around $4.5 \%$ of MS cases are late onset. Scarcely ever, it affects people older than 60 years. Commonly observed characteristics of LOMS include primary progressive clinical course, more common involvement of Spinal cord and a rarity of Gadolinium enhancing lesions [1,2]. The case presented below, however, depicts quite a deviation and shows how divergent LOMS might be in its presentation, Imaging findings and prognosis.
\end{abstract}

Keywords: Multiple sclerosis; Late Onset MS; EDSS scale

Abbreviations: LOMS: Late Onset Multiple Sclerosis, CSF: Cerebrospinal Fluid; MRI : Magnetic Resonance Imaging, EDSS: Expanded Disability Spectrum Scale

\section{Introduction}

Late Onset Multiple Sclerosis is defined as symptom onset at or after the age of 50 years. When it starts at or after the age of 60 , it is referred to as Very late onset MS [3,9]. As most of the studies done regarding clinical, radiological and prognostic features of MS, in past, have usually excluded patients older than 50 or 55 years of age, very little is known about behaviour of the LOMS [4]. Nevertheless, it is generally regarded as more rapidly debilitating condition than young onset relapsing remitting multiple sclerosis [7] (YOMS). Motor symptoms are the most common initial presentation, followed by sensory symptoms, fatigue and sphincteric disturbances. Diagnosing MS in older people is also challenging as clinical features are quite nonspecific and can be attributable to other common comorbidities of that age. Imaging may be confusing as demyelinating lesions of neurons in brain may be wrongly thought of as white matter changes due to small vessel disease, or sometimes lymphomas [5]. Moreover, spinal degenerative changes also make clinicians hesitant of attributing symptoms to the spinal cord's demyelinating pathology [6]. The given case adds to the colours of demographic and clinical spectrum of LOMS. It is interesting in many ways, due to its unique nature.

\section{Case Report}

The case is about a 63-year-old lady of Pakistani origin, who presented to us in July 2016 (61 years old at that age).
Only significant medical comorbidities were well controlled Hypertension on lisinopril, and hypothyroidism on thyroxine. Presenting complaint was headache and a four month history of weakness in left lower which improved significantly but did not return to baseline. She had no visual problems in past, though she agreed to having fatigue, pins and needles sensation in both hands and some features of urge incontinence .There was no history of febrile illness or weight loss.. Examination showed equal response to light from both pupils, normal range of extra ocular movements, no nystagmus, intact coordination, only positive finding was mild spasticity of left leg along with mild weakness of left foot dorsiflexion.

MRI BRAIN was done which showed multiple T2 hyperintense corpus callosal and periventricular lesions with their long axis perpendicular to the long axis of the lateral ventricles, One lesion in white matter corresponding to right precentral gyrus was relatively large, rounded with significant perilesional edema I, and showed faint rim enhancement ( not the typical open ring of tumefactive lesions facing grey matter) MR Spectroscopy was performed which showed increase in choline but not in creatine, and no reduction in NAA. No lactate peak was observed in any of lesions. Cervicothoracic Spine MRI was a normal study. Overall picture was favouring Multiple Sclerosis (meeting the 2017 McDonald's criteria of one clinical attack and imaging evidence of dissemination in space), so she was convinced for further investigations. 


\section{Open Access Journal of Neurology \& Neurosurgery}

Nerve conduction studies were done for both lower limbs which showed normal motor and sensory conduction velocities for both sural, tibial and common peroneal nerves. Visual evoked potentials were done which showed normal latencies for both eyes, $\mathrm{p} 100=106 \mathrm{~m} \mathrm{sec}$ for left and $107 \mathrm{~m} \mathrm{sec}$ for right eye (Figure 1).
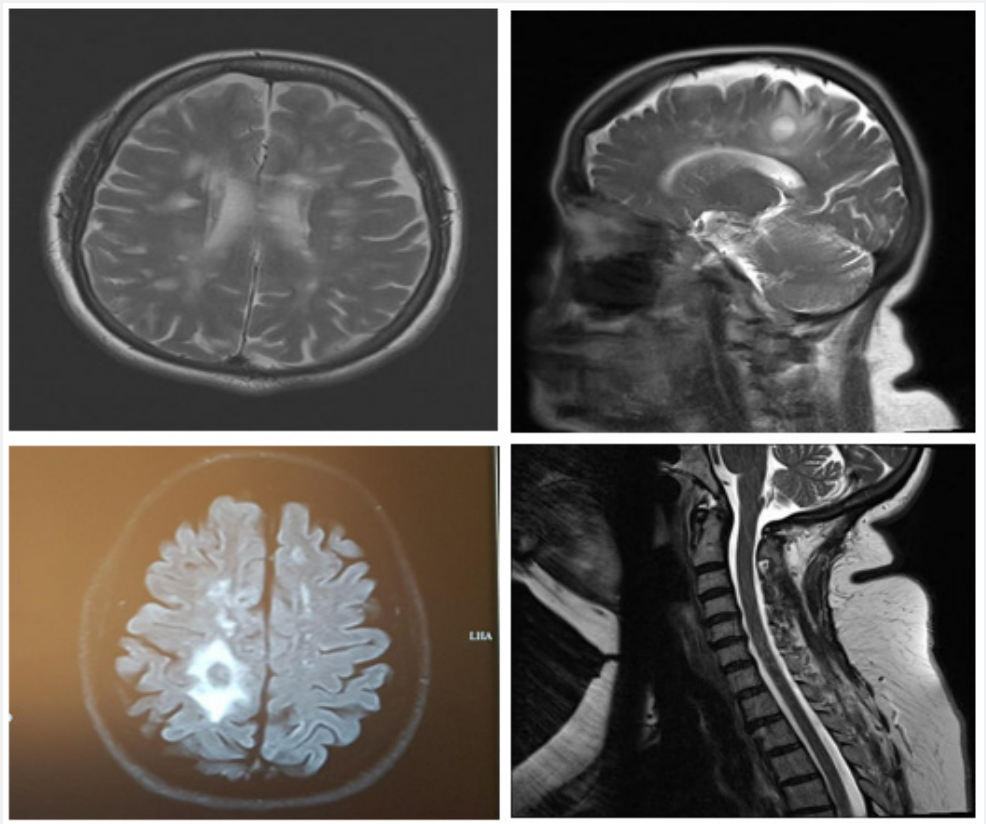

Figure 1: MRI spine sagittal T2-weighted images of the cervical spine (left) and lower thoracic spine (right).

Lumbar puncture was done, CSF study demonstrated normal WBC counts and protein, one sample of CSF and serum was sent for Isoelectric focusing. It showed a type 2 profile, i.e. presence of oligoclonal IgG in CSF and absent from serum depicting intrathecal immunoglobulin synthesis, further consolidating the diagnosis of MS. Extensive blood workup was done to rule out mimics of multiple sclerosis, like Neurosyphilis, Sarcoidosis,
Vasculitis, metastases etc. VDRL, HIV screen, Rheumatoid factor, ANA, AntiDs DNA, Anti phospholipid antibodies, Vitamin profile and serum copper, Serum protein electrophoresis, Complement levels, Cryoglobulins and tumour markers. All these tests failed to provide an alternative diagnosis. CT Chest Abdomen and Pelvis did not reveal any significant abnormality.
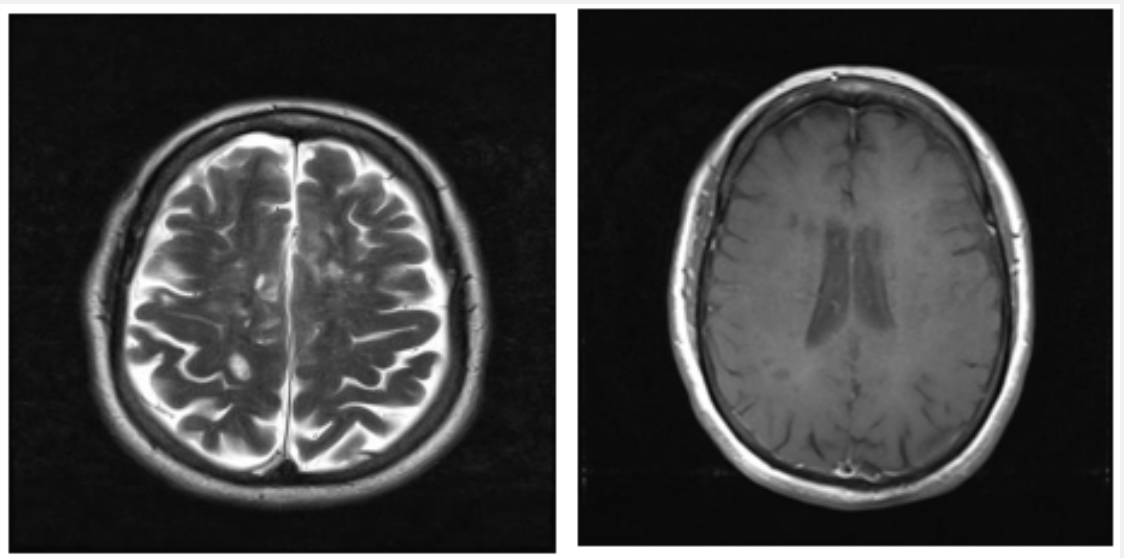

Figure 2: Repeat scan after six months showing resolution of edema and marked decrease in size presulcal T2 lesion (right), no contrast enhancement was seen (left).

She was given a three day pulsed IV Methylprednisolone trial in view of edema seen around the larger right periventricular lesion, followed by a tapering course of oral prednisolone. She improved almost completely in terms of weakness and urge incontinence though fatigue remained at a milder level. Option of getting long term Disease Modifying Therapy (DMT) was not favoured by her. Repeat MRI BRAIN after six months (01/2017) did not show any noticeable interval change except decrease in size of the pre sulcal right periventricular lesion, with total resolution of the surrounding edema. There was no contrast 


\section{Open Access Journal of Neurology \& Neurosurgery}

enhancement. Matter of DMT initiation was brought into discussion but patient was not keen at that moment (Figure 2).

On further follow up after six months (08/2017), she gave an account of right sided numbness and heaviness involving both upper and lower limbs which resolved completely in few days. However, her left foot dorsiflexion weakness had returned and persisted. A fresh MRI BRAIN was done and compared with her last MRI BRAIN scan Two new lesions were seen in left corona radiata, one showed Gadolinium enhancement. With this new relapse, not only Macdonald's Criteria were further satisfied (2010 criteria) but relapsing remitting nature of her disease was confirmed. This time she was ready to receive disease modifying therapy. Due to her low EDSS of 2.5, she was started on Interferons (weekly intramuscular injections of interferon beta-1a) [9] (Figure 3).
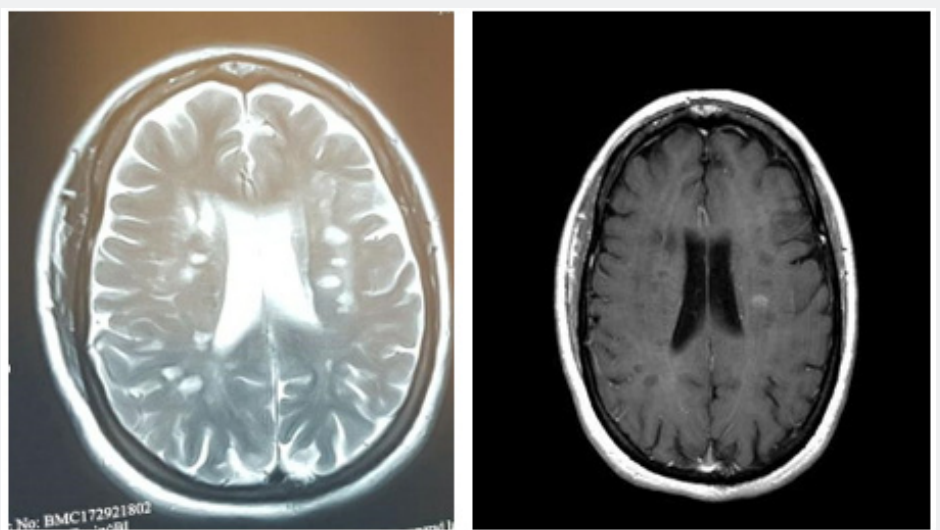

Figure 3: A further follow up scan demonstrating interval appearance of two new T2 hyperintense lesions in left corona radiata (compare with fig.1) one shows gadolinium enhancement (left).

She remained stable on interferon treatment, until in January 2019 when she presented with weakness of right lower limb and gait instability. Exam showed spasticity and mild weakness of 4/5 in right hip and knee flexion with hypertonia. She was pulsed with methylprednisolone and weakness totally resolved. MRI Brain with contrast showed a new but non enhancing T2 hyperintense lesion in left anterior corpus callosum bulging into the left lateral ventricular cavity. This lesion showed mild restriction on DWI but no gadolinium enhancement. She was planned for upgradation of therapy to fingolimod, but she had recently diagnosed to be diabetic, Natalizumab was out of favour due to JC virus positivity. Finally, after mutual agreement she has been started on teriflunomide. At the time of this writing, her EDSS score is stable at 2.5 (Figure 4).

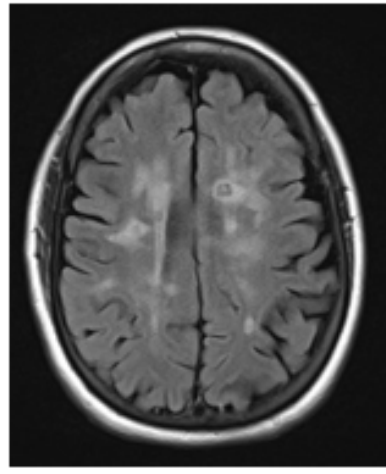

(a)

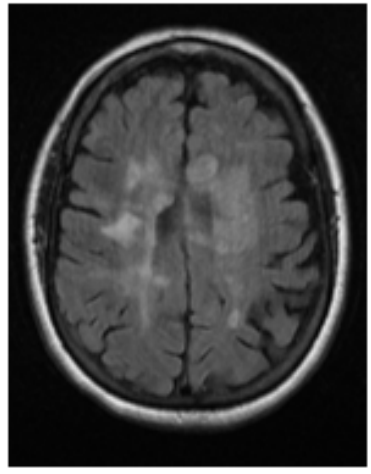

(b)

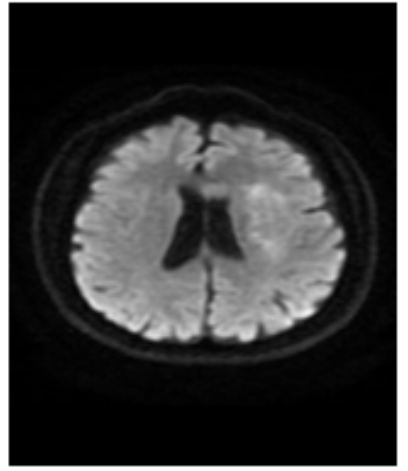

(c)

Figure 4: A Follow up scan (b) showing a new T2 hyperintense lesion (flair sequence) in left anterior corpus callosum bulging into the left lateral ventricular cavity; mildly restricting on DWI. Fig (a) Shows old T2 flair scan from 2017.

\section{Discussion}

This case is a good example of the multifarious spectrum of Late onset MS. Although the presenting symptom was that of a motor domain, other features were not what have usually been associated with LOMS [2-4]. For example, clinical course was relapsing remitting, and there was no accumulation of disability, though the period of follow up was only two years.
EDSS remained stable at around 2. Furthermore, MRI Brain did not show cerebellar or infratentorial lesions, Spinal cord was not affected, and Gadolinium enhancement was observed on two occasions [6]. All these characteristics are usually less expected from the late onset MS, as seen in multiple studies [1-6]. This implies that, as more and more cases of MS in older people are reported from different parts of the world, the perception of late onset disease as a more gruelling disorder may change. 


\section{References}

1. Martinelli V, Rodegher M, Moiola L, Comi G (2004) Late onset multiple sclerosis: clinical characteristics, prognostic factors and differential diagnosis. Neurol Sci 25 (Suppl 4): S350-355.

2. Kis B, Rumberg B, Berlit P (2008) Clinical characteristics of patients with late-onset multiple sclerosis. J Neurol 255(5): 697-702.

3. Roohani P, Emiru T, Carpenter A, Luzzio C, Freeman J, et al. (2014) Late onset multiple sclerosis: Is it really late onset? Mult Scler Relat Disord 3(4): 444-449.

4. Shirani A, Zhao Y, Petkau J, Gustafson P, Karim ME, et al. (2015) Multiple Sclerosis in Older Adults: The Clinical Profile and Impact of Interferon Beta Treatment. Biomed Res Int 2015: 451912.

5. De Seze J, Delalande S, Michelin E, Gauvrit JY, Mackowiak MA, et al (2005) Brain MRI in late-onset multiple sclerosis. Eur J Neurol 12(4): 241-244.

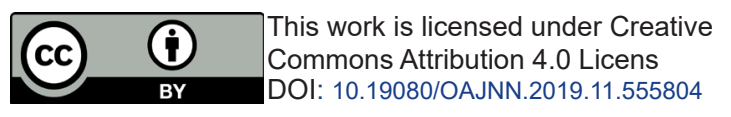

6. Bermel RA, Rae-Grant AD, Fox RJ (2010) Diagnosing multiple sclerosis at a later age: more than just progressive myelopathy. Mult Scler 16(11): 1335-1340.

7. Leary SM, Porter B, Thompson AJ (2005) Multiple sclerosis: diagnosis and the management of acute relapses. Postgrad Med J 81(955): 302308.

8. Rice CM, Cottrell D, Wilkins A, Scolding NJ (2013) Primary progressive multiple sclerosis: progress and challenges Claire. J Neurol Neurosurg Psychiatry 84(10): 1100-1106.

9. ED Ciftci, S Kibaroglu, US Benli (2013) A case of very late onset multiple sclerosis. 333(Supl 1): 382.

\section{Your next submission with Juniper Publishers will reach you the below assets}

- Quality Editorial service

- Swift Peer Review

- Reprints availability

- E-prints Service

- Manuscript Podcast for convenient understanding

- Global attainment for your research

- Manuscript accessibility in different formats

( Pdf, E-pub, Full Text, Audio)

- Unceasing customer service

Track the below URL for one-step submission

https://juniperpublishers.com/online-submission.php 